\title{
Design and Implementation of Spatial Interweave LTE-TDD Cognitive Radio Communication on an Experimental Platform
}

\author{
Boris Kouassi, Bassem Zayen, Raymond Knopp, Florian Kaltenberger, Dirk Slock, Irfan Ghauri, \\ Francesco Negro, Luc Deneire
}

\begin{abstract}
Cognitive radio, which enables smart use of wireless resources, is a key ingredient to achieve high spectral efficiency. LTE, the latest evolution of cellular standards, is widely adopted and also targets high spectral efficiency. Hence, to enable wide adoption of cognitive radio, using LTE as the physical layer is a natural choice. Targeting a real-time implementation of LTEbased cognitive radio, we focus on spatial interweave cognitive radio, in which a secondary user uses an antenna array to perform null-beamforming in the primary user's direction, hence reusing the spectrum spatially. To allow this, without any help from the primary system, we use the time division duplex (TDD) mode and take advantage of the channel reciprocity. However, this reciprocity is jeopardized by the mismatch between the RF front-ends. Hence, we design a calibration protocol to restore it. The whole system is implemented and evaluated on EURECOM's experimental OpenAirInterface platform. Performance results are presented, showing the feasibility of spatial interweave cognitive radio on a real-time platform.
\end{abstract}

Index Terms-Spatial interweave cognitive radio, beamforming, channel estimation, reciprocity calibration, LTE-TDD platform.

\section{INTRODUCTION}

Cognitive radio (CR) approaches [1] are increasingly highlighted due to the concern for radio spectrum optimization. This concern is generated by the accelerated evolution of radio technologies and the ensuing spectrum unavailability. CR approaches have been classified in three main groups: underlay CR which supposes simultaneous transmissions but under an interference (temperature) limit, overlay CR which introduces a cooperation concept, and interweave $\mathrm{CR}$ in which secondary transmissions are achieved by exploiting the white spaces in primary spectrum see [2]. In the interweave paradigm, the primary white spaces can be observed in time, frequency or space dimension [3].

B. Kouassi and L. Deneire (\{kouassi, deneire $\} @ i 3 s . u n i c e . f r)$ are with the laboratory I3S at the University of Nice Sophia Antipolis, France. B. Zayen, R. Knopp, F. Kaltenberger, F. Negro and D.T.M. Slock (\{zayen, knopp, kaltenberger, negro, slock\}@eurecom.fr) are with Mobile Communications Department of EURECOM, Sophia Antipolis, France. I. Ghauri (ghauri@intel.com) is with Intel Mobile Communications, Sophia Antipolis, France.

EURECOM's research is partially supported by its industrial members: BMW Group Research \& Technology, Swisscom, Cisco, ORANGE, SFR, STMicroelectronics, Thales, Symantec, Monaco Telecom and by the EU FP7 FET project CROWN and STREP projects SACRA and WHERE2.

The research of Intel Mobile Communications is also supported in part by the EU FP7 projects CROWN and SACRA

\section{A. Cognitive Radio Implementations}

$\mathrm{CR}$ technologies have been heavily investigated this last decade, leading to both large standardization and implementation efforts. Several working groups have been created for CR standardization (see e.g [4] and references therein), for instance the IEEE 802.22 working group for the wireless regional area network (WRAN) CR standard. This standard aims at using white spaces in the unused television allocated spectrum for CR technologies. Some other standards have been developed like the IEEE SCC41 (Standards Coordinating Committee 41) for dynamic spectrum access networks. Due to the growing interest in CR applications, several telecommunication companies and operators focus their research and development efforts on CR technologies. Consequently, many projects and experimental platforms have been designed to implement CR, e.g the Canadian CORAL (CR learning platform) project based on WiFi (IEEE $802.11 \mathrm{~g} / \mathrm{a}$ ), the hardware platform USRP (universal software radio peripheral), and the complete hardware/software WARP (wireless open-access research) platform, a CR system designed by the Rice University [5]. Furthermore, the literature illustrates other platforms (see e.g [5] and references therein), and most of the developed platforms are based either on non-standard PHY/MAC layers or on $\mathrm{WiFi}$, e.g. targeted to non-licensed bands.

Our approach, the design of a cognitive radio system in the LTE framework, enables the deployment of CR in licensed bands, with no hardware modification and only small software changes in some specific frames for the secondary system.

\section{B. LTE as the global standard}

LTE (Long Term Evolution) is the latest cellular networks standard, developed and supported by 3GPP and 3GPP2, is currently under deployment in Europe, and is deployed since 2009-2010 in Japan and in USA (with more than 16 million subscribers for Verizon only at the end of 2012). This wide support from standardization bodies and world industry means that LTE-Advanced, the next step after LTE, is "the" global standard for the $4^{\text {th }}$ generation cellular networks. LTE is a "near 4G" standard, providing downlink (DL, from base station to mobile) rates up to $300 \mathrm{Mbit} / \mathrm{s}$, uplink (UL, from mobile to base station) rates up to $75 \mathrm{Mbit} / \mathrm{s}$, in a $20 \mathrm{MHz}$ bandwith, using $4 \times 4 \mathrm{MIMO}$ and a round trip time of $10 \mathrm{~ms}$, whereas $4 \mathrm{G}$ targets $1 \mathrm{Gbit} / \mathrm{s}$ in the UL (100 Mbit/s in high mobility) in $100 \mathrm{MHz}$ bandwith and using $8 \times 8 \mathrm{MIMO}$. The 
modulation is OFDMA in the DL, and Single Carrier-FDMA in the UL. LTE uses frequency division duplex (LTE-FDD) as well as time division duplex (LTE-TDD).

\section{LTE-TDD and spatial interweave}

Different CR implementations based on the TDD are reported in the literature. In these implementations, the UL and DL transmissions are performed in the same frequency band (see e.g. [3], [6]). Consequently, in a TDD, the UL/DL propagation channels are reciprocal and this reciprocity is exploited in particular in the spatial interweave (IW) CR approach adopted in this paper. In IW-CR, reciprocity helps to overcome several constraints like the channel estimation at the transmitter (Tx), and more importantly lack of cooperation! [3], [6]. Specifically, a persistent problem in spatial IW-CR is to find the best way for secondary users (SUs) to identify and then to transmit in the primary spatial white spaces, without disturbing the primary users (PUs). This can be done by beamforming (linear precoding). Beamforming uses an antenna array and SU-to-PU channel state information at the Tx (CSIT) to mitigate the interference towards the primary receiver $(\mathrm{Rx})$ and/or steer a signal towards a specific secondary Rx. Thereby, it maximizes the signal to interference plus noise ratio (SINR) for the considered receiver [7]. Normally, beamforming at the Tx is achieved using a feedback procedure. Because we suppose no cooperation between the primary Rx and the secondary Tx, the feedback is not an option. However the feedback constraint can be overcome by exploiting the UL/DL channel reciprocity in TDD mode. Nonetheless as previously mentioned in [8], [6], [9], the UL/DL channel reciprocity only applies to the propagation part, and not to the overall digital channel response due to radio frequency (RF) circuit mismatches. Additional studies have proposed calibration solutions to compensate the RF impairments and make the channel reciprocity a reality in practice [9], [6].

Despite the importance of calibration for future wireless communication, few algorithms exist in the literature. The "relative" calibration has been selected in this paper. Unlike the "absolute" calibration it does not require any equipment and compensates the non reciprocal RF using the relation between the UL/DL channels. Subsequently, the authors in [9] assume that the calibration parameters are invariant during a long period (typically seconds). This assumption allows to exploit several versions of the UL/DL channels over the time, and to derive the calibration parameters through a total least squares (TLS) formulation [10]. However, a frequency offset can occur in the consecutive channel estimations. Thereby, authors in [11] illustrate a calibration scheme which automatically compensates for the offset. We adopt the same calibration framework as presented in [6], [12], [8]. Specifically, we determine the calibration factors by subdividing the multiinput multi-output (MIMO) channel into single channels [13].

However, whereas relative calibration requires a signaling between Tx and Rx, no cooperation from the PUs can be expected in the IW paradigm. This is where "any-com $T x$ calibration" comes in: Tx calibration by any-link communication [3]. This concept appears to be reused in the Argos calibration proposal for Massive MIMO [14]. In IW-CR, the SU-Tx can be calibrated by communicating with the SU-Rx, obviating the need for any cooperation from the primary.

\section{OpenAirInterface Platform}

OpenAirInterface (www.openairinterface.org) is an opensource software defined radio platform for experimentation in wireless systems with a strong focus on cellular technologies such as LTE and LTE-Advanced. The current OpenAirInterface software modem comprises a highly optimised C implementation of all the elements of the 3GPP LTE Rel 8.6 protocol stack (PHY, MAC, RLC, RRC, PDCP) for both UE and eNB. Transmission modes 1 (SISO), 2 (Alamouti), 5 (MUMIMO), and 6 (beamforming) are currently supported. The software modem can be run in simulation/emulation mode or in real-time mode together with a hardware target such as ExpressMIMO. This card currently supports up to two antennas and a bandwidth of $5 \mathrm{MHz}$. A flexible radio frequency frontend supporting carrier frequencies from $300 \mathrm{MHz}$ up to $3.8 \mathrm{GHz}$ and transmission powers up to $30 \mathrm{dBm}$ is also available.

The rest of the paper is structured as follows. Section II describes the beamforming and TLS-based calibration. In Section III we detail the LTE-TDD parameters. The spatial IW implementation using OAI is described in Section V and IV, while performance evaluation is presented in SectionVI. Finally, the conclusions are summarized in Section VII.

\section{Reciprocity-based Spatial IW-CR SCEnARIO}

\section{A. The System}

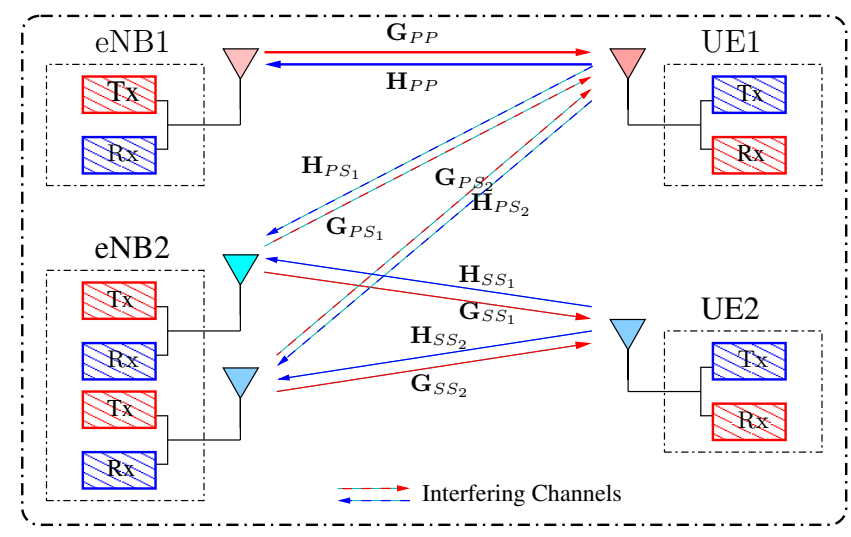

Fig. 1. Illustration of the primary system including eNB1 and UE1, and the secondary system, eNB2 and UE2, with interfering channels between systems.

Fig. 1 presents the CR scenario with a primary system including the licensed users, eNB1 and UE1 designed with one antenna, and a secondary system composed of opportunistic users, eNB2 and UE2 with two antennas and one antenna respectively, which are not licensed to transmit in the radio environment. The denomination eNodeB (eNB) and user equipment (UE) is conform to LTE, and depicts a communication including one node and an attached LTE-TDD equipment. The radio frequency circuitry (RF) is represented on Fig. 1 by the transmission (Tx) and reception (Rx) filters for each antenna. Throughout the paper, we use the following notation (where all quantities are in the frequency domain) : 
- $\mathbf{G}_{S S_{i}}$ is the DL channel gain between $i$-th Tx antenna at eNB2 and Rx antenna at UE2, and $\mathbf{H}_{S S_{i}}$ the UL channel gain between Tx antenna at UE2 and $i$-th Rx antenna at eNB2;

- $\mathbf{G}_{P P}$ is the DL channel gain between UE1 Rx and eNB1 $\mathrm{Tx}$, and $\mathbf{H}_{P P}$ the UL channel gain between UE1 Tx and eNB1 Rx;

- $\mathbf{G}_{P S_{i}}$ is the DL channel gain between $i$-th Tx antenna at eNB2 and Rx antenna at UE1, and $\mathbf{H}_{P S_{i}}$ the UL channel gain between Tx antenna at UE1 and $i$-th Rx antenna at eNB2;

- $\mathbf{C}_{S_{i}}$ is the calibration factor between $i$-th eNB2 antenna and UE2 antenna.

We assume a multipath channel, then the OFDM configuration from LTE leads to decompose the frequency selective channel into several parallel simple (additive white Gaussian noise) AWGN channel on sub-carriers in frequency domain. In this study, the spatial interweave CR system consists in mitigating in DL transmissions, the interference from the SU transmitter (eNB2) to the PU receiver (UE1). The proposed interference avoidance technique is a simple zero forcing beamforming (ZFB) technique implemented in SU base station eNB2.

\section{B. Initial Beamforming Approach}

We aim at sending a null interference in the direction of UE1, leading to the relation:

$$
\begin{aligned}
\mathbf{y}_{p} & =\mathbf{G}_{p p} \mathbf{x}_{p}+\mathbf{G}_{p s} \mathbf{x}_{s}+\mathbf{n} \\
\mathbf{G}_{p s} \mathbf{p s}_{x} & =\left[\begin{array}{ll}
g_{p s 1} & g_{p s 2}
\end{array}\right]\left[\begin{array}{l}
p_{1} \cdot s_{x 1} \\
p_{2} . s_{x 2}
\end{array}\right]=0,
\end{aligned}
$$

with $\mathbf{y}_{p}$ the per-subcarrier received signal at the primary user UE1, $\mathbf{n}$ the frequency domain AWGN introduced at the PU receiver, and $\mathbf{G}_{p s} \mathbf{x}_{s}$ the interference generated by the secondary transmitter eNB2. We design a precoder (beamformer) $\mathbf{p}$ at eNB2 such that $\mathbf{G}_{p s} \mathbf{x}_{s}=0, \mathbf{x}_{s}=\mathbf{p} \mathbf{s}_{x}$, where $\mathbf{s}_{x}$ is the transmitted symbol at eNB2. We design $\mathbf{p}$ from the crosslink channel coefficient $\left(\hat{\mathbf{G}}_{p s}=\left[\begin{array}{ll}\hat{g}_{p s 1} & \hat{g}_{p s 2}\end{array}\right]\right)$. Thereby, the efficiency of the precoder $\mathbf{p}$ will be related to the reliability of the crosslink channel estimation. This channel cannot be estimated directly by the secondary eNB2, because cooperation between primary and secondary systems is not allowed. But, using the channel reciprocity assumption, we can theoretically find the DL channel using only the UL channel.

However, in order to avoid crosslink reciprocity-calibration between PUs and SUs, we show in Section IV how the calibration parameters at the secondary transmitters allows to estimate the crosslink DL channel without any feedback from the PUs. Thus, we obtain information required for Tx beamforming through smart exploitation of received signal from PUs during a TDD frame.

\section{LTE PARAMETERS USED}

The $10 \mathrm{~ms}$ periodic TDD frame type 2 with the configuration number 3 is used in this study (see Fig. 2) [15]. It is composed of 10 subframes with the OFDM multiplexing in the UL and the DL. Each subframe is divided into 2 time slots (TS). A TS is consisting of 7 OFDM symbols and an extended cyclic prefix to avoid inter symbol interference.

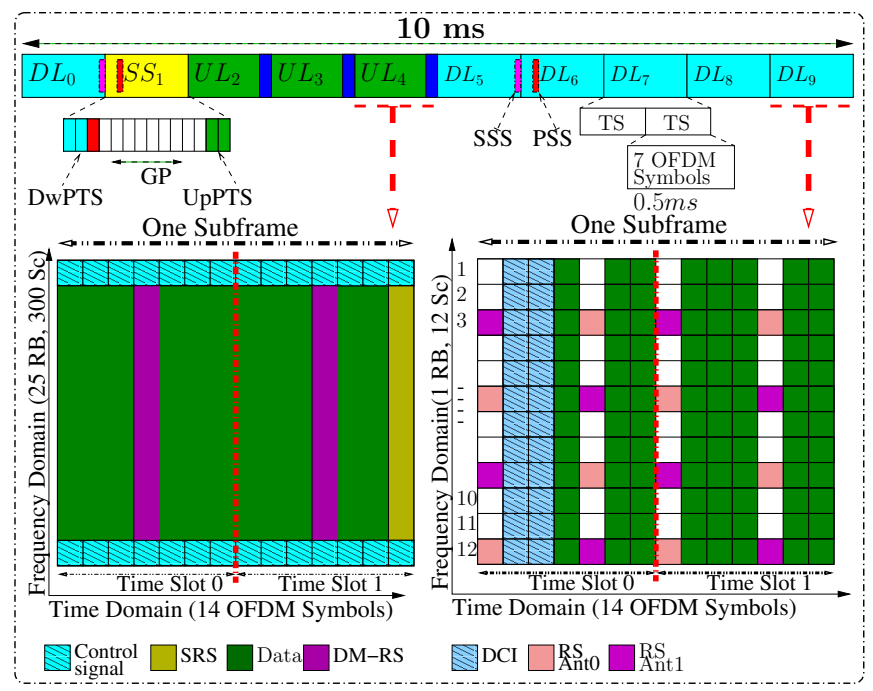

Fig. 2. TDD Frame specifications, with the synchronization signals (PSS/SSS), the guard period (GP), and the DL/UL pilot time slot Dw/Up-PTS. The extension in the DL shows a resource block (12 subcarriers $(\mathrm{Sc})$ and 2 TS) with a normal cyclic prefix, the pilots locations, the control signaling and data location. In the UL, we observe the $300 \mathrm{Sc}$ subframe structure.

\section{A. Downlink (DL) Subframe}

Fig. 2 describes the DL subframe with RSs locations in OFDM symbols for 2 transmit antennas at eNB2. The RSs from different antennas are located in specific resource elements (RE) to ensure the orthogonality. Therefore, REs including the pilots in RBs are not used for data transmissions, and the transport block size depends on the number of antennas. The first and third RSs are related to antenna 0 and the second and the fourth to antenna 1 (see Fig. 2). The first TS of each subframe is consisting of the DL control information which carries signaling information (resource block assignment, modulation and coding scheme, etc).

\section{B. Uplink (UL) Subframe}

As can been observed in Fig. 2, in the uplink, control signals (CS) fill the RBs borders. The RSs used for UL signal demodulation (demodulation RS: DM-RS) and those for channel quality estimation (sounding RS: SRS) are located in specific symbols between CSs. The data fills the remaining resource elements and are associated with DM-RS on physical uplink shared channel (PUSCH). Because of limited resources and constraints in the UL, the control signaling is different with the DL case, and is mainly located at the eNB side. The eNB has a prior knowledge about control information of UL data, and carries a large part of the signaling, which alleviates the overhead at the UEs.

\section{Special Subframe (SS)}

The Special Subframe (SS) described in Fig. 2 separates DL and UL transmissions. Specifically, the guard period (GP) is the switching point, and determines the maximum supportable cell size. The DwPTS is treated as a regular but shortened DL subframe, it always contains RS and control information like a regular DL subframe, and may transmit data. In addition, in the TDD-LTE frame, it also contains the primary DL synchronization signal (PSS). 


\section{SPatial InterweaVe (IW) IMPLEMEntation}

\section{A. Spatial IW Beamforming}

The goal is to achieve spatial interweave based only on secondary communication (eNB2) without collaboration from PUs. The overall DL channels (including the RF parameters) in secondary-to-secondary link $\left(\mathbf{G}_{S S}\right)$, and in the secondaryto-primary link $\left(\mathbf{G}_{P S}\right)$ are defined in relation (2). Then, the information provided by the crosslink channel $\mathbf{G}_{P S}$ is exploited to design the reciprocity-based beamformer. The spatial IW beamformer $\mathbf{p}$ in Section II-B, allows to write

$$
\mathbf{G}_{S S}=\mathbf{C}_{M_{S}} \mathbf{H}_{S S}^{T} \mathbf{C}_{B_{S}} ; \mathbf{G}_{P S}=\mathbf{C}_{M_{P}} \mathbf{H}_{P S}^{T} \mathbf{C}_{B_{S}}
$$

$\mathbf{G}_{P S} \mathbf{p}=\left(\mathbf{C}_{M_{P}} \mathbf{H}_{P S}^{T} \mathbf{C}_{B_{S}}\right) \mathbf{p}=0 \Leftrightarrow\left(\mathbf{H}_{P S}^{T} \mathbf{C}_{B_{S}}\right) \mathbf{p}=0$, with $\mathbf{C}_{B}$ and $\mathbf{C}_{M}$ respectively the calibration matrices at eNB and UE. We observe that this solution is insensitive to $\mathbf{C}_{M_{P}}$, the matrix at UE1. Hence, the eNBs matrix $\mathbf{C}_{B_{S}}$ for transmission to the primary can be derived from the secondary-to-secondary calibration and without feedback from the primary system.

The required calibration parameter is the same in the secondary-to-secondary link as in the secondary-to-primary link, thereby secondary calibration suffices to compute the beamformer. We define a first training step where the eNB2 estimates the interfering UL channel with the UE1. Then, the beamformer $\mathbf{p}$ is applied in the baseband processing on each eNB2 OFDM subcarrier (see Fig. 3).

\section{B. Reciprocity-Calibration in $O A I$}

We will adopt the calibration technique described in [13] and evaluated in [6] and [12]. Then the problem boils down to subdividing the multi-input single-output (MISO) SU DL channel into 2 single channels, and solved using a total least squares (TLS) formulation (see [6]).

From the study [6], we observe that $K$ subsequent versions of UL/DL channels are required to increase the accuracy of calibration in noisy channels. Therefore, an initial short feedback is achieved.

We suppose the channel constant for at least one frame duration. Then, for each subcarrier, $K$ estimations of UL and their corresponding DL channels are saved sequentially in a buffer for each frame (10ms, 300 subcarriers).

For the sake of simplicity, we write the RF parameters of UE and eNB in the same variable $\mathbf{C}_{S}$ such $\mathbf{G}_{S S}=\mathbf{C}_{S} \mathbf{H}_{S S}^{T}$, since we use 1 antenna at the secondary UE. Then, assuming similar RF perturbations at users side, we use the singular value decomposition (SVD) solution of TLS problems presented in [10]. For each single subcarrier, the calibration factors will be represented by a scalar value $c_{i, j}$, the factor for antenna $i$ and subcarrier $j$. After the calibration, the eNB2 is able to estimate the DL crosslink channel coefficients just by using the UL and the calibration parameters.

\section{OAI SOFTWARE IMPLEMENTATION}

The proposed spatial interweave method has been implemented and integrated into the OpenAirInterface software modem. In this paper we present results from simulations, which allow to test the algorithms in a controlled environment i.e., primary and secondary system are perfectly synchronized and we can experiment with different channel models. The same code equally runs in real-time on the hardware target.

\section{A. Restoring reciprocity}

We introduce in Fig. 3 the decalibration filters and a frequency offset variation in order to simulate the effects of RF impairments. The final goal is to restore the channel reciprocity (despite these perturbations) and to improve the transmission using the spatial IW precoding at eNB2 Tx. In this paper we suppose a low frequency offset with no significant impact on the transmissions. We assume different

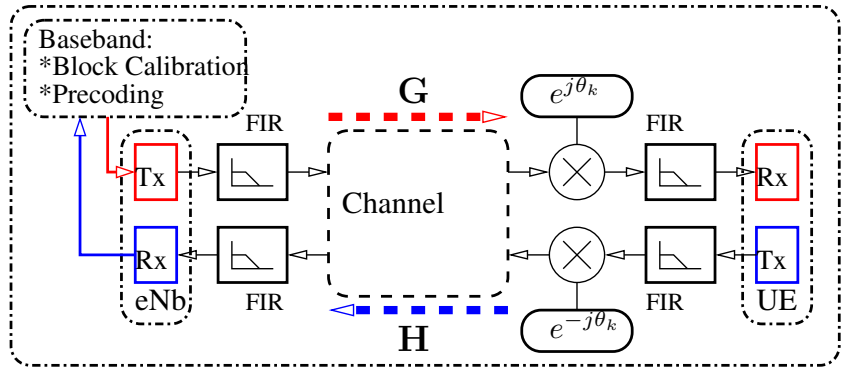

Fig. 3. Illustration of simulation chain and software implementation. Effects of RF front-ends are modeled using finite impulse response filters (FIR) and phase drifts are simulated using the exponential.

channel models, and using the TDD frame structure in Fig. 2, the number of frames for the experiment is set to 500 , and the number of frames for calibration to 15 based on [6]. The idea is to transmit the calibration pilots from eNB2 to UE2 in SS, then to start the feedback of DL channel coefficients, since the PUs are not transmitting in SS. The corresponding UL/DL channel are saved at the eNB2, which in turn computes the calibration factors. These calibration factors are used to infer the crosslink DL channel from the crosslink UL channel. Then the ZFB is computed at the eNB2 using the crosslink DL channel (see Section IV-A, II-B). Because they depend on electronics, calibration factors vary slowly (seconds or more). The implementation procedure is summarized in the following algorithm:

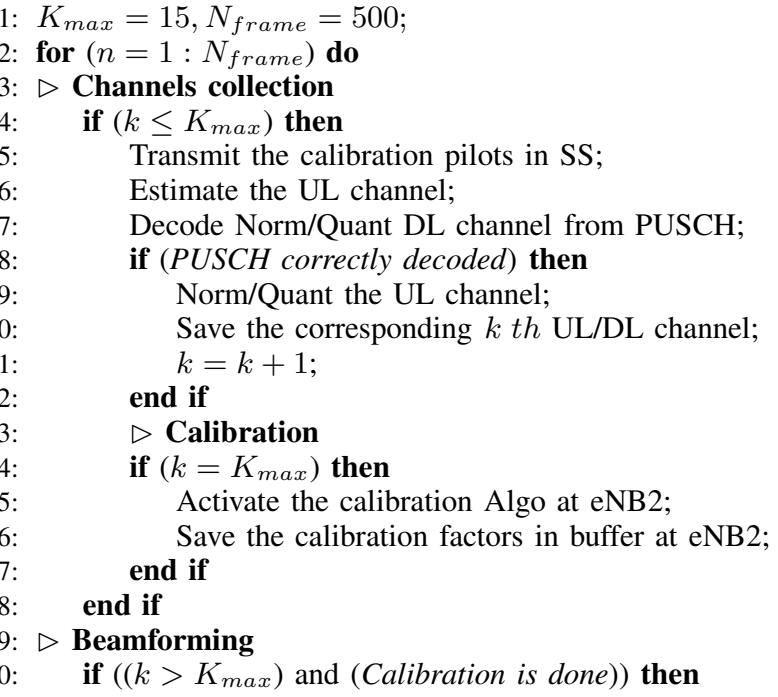




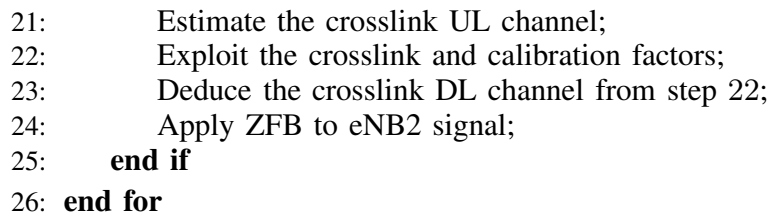

\section{B. Tweaking LTE's Special Subframe}

In order to fit the scenario in Fig. 1, we assume that the SUs are aware of PUs specifications and use the same configuration. We use the LTE transmission mode 1 , because only one antenna is used at PUs. The problem is that channel estimations from two antennas of eNB2 are mandatory for the calibration algorithms. We propose to modify the initial LTE special subframe (SS) structure in SUs, while avoiding to perturb the conventional PUs LTE transmissions. Fig. 4 shows the new positioning of the RS (cell-specific RS) in SS. The considered location of the REs is exceptionally designed for 2 antennas channel estimation in transmission mode 1 . The new location of RS occupies 1 OFDM symbol, and holds in the SS. Note that the RS of ant 0 and ant1 (see Fig. 4) are spread in frequency and are delayed by 2 subcarriers. Upon reception, the overall channel estimates are obtained by interpolation (see e.g. [15]) adapted to the new RS positions in the SS. The next section describes the channel estimation procedure.

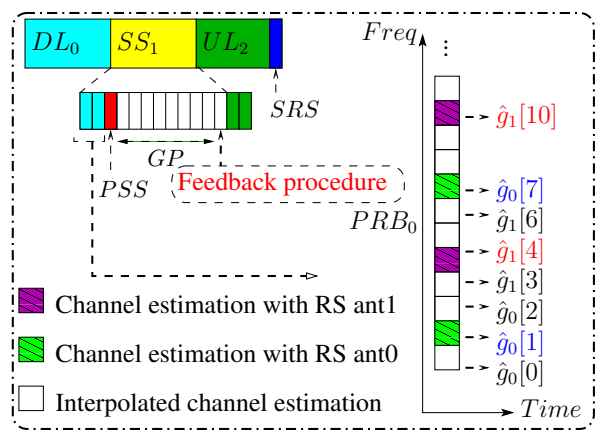

Fig. 4. Illustration of the special subframe (SS) structure showing the exploitation of SS for feedback process. The channel estimation from reference signals (RS) is estimated by a least squares approach. In the empty locations, channels are estimated using an interpolation (or extrapolation) procedure.

\section{Channel Estimation in Frequency Domain}

The channel estimation procedure is based on training sequences (RS). As described in Fig. 4, special pilots for antennas 0 and 1 are transmitted orthogonally in specific resource elements on the first OFDM symbol in SS. These locations are known at the TX/Rx side. At the RX, the SU first estimates independently the channel coefficients for each corresponding subcarriers in the first symbol.

Let's $P_{i}[j]$ denote for the frequency domain pilot designed for the antenna $i$ on the $j$ th subcarrier. The frequency domain DL channel coefficient $\hat{g}_{i}[j]$ (antenna $i$ on subcarrier $j$ ) estimated by SU where there is a predefined RS (see Fig. 4) is found using a least squares approach. The channel coefficients in white resource elements (REs) see Fig. 4 (i.e. without any predefined pilot location) are estimated using already estimated channels and linear interpolation or extrapolation.

\section{TRANSMission PARAMETERS AND NUMERICAL RESULTS}

\section{A. Transmission Parameters}

We aim at defining the beamforming at the SUs without the cooperation of PUs. Thus the bulk of algorithms and complexity are centralized in eNB2, while the UE2 estimates and feeds the DL channel back for calibration. The realtime transmission is achieved assuming a sample duration $T_{s}=130.2 n s$, with an OFDM symbol duration of $71.35 \mu \mathrm{s}$ $\left(548 T_{s}\right.$, including a cyclic prefix length $\left.36 T_{s}, 4.69 \mu \mathrm{s}\right)$ and a 15 $\mathrm{kHz}$ subcarriers (subcarrier) spacing. We use a central carrier frequency equal to $1.9 \mathrm{GHz}$, the bandwidth is divided into 25 Resource Blocks (RB) of 12 subcarriers each. The OFDMA configuration in DL yields to a maximum Tx bandwidth up to $4.5 \mathrm{MHz}(12$ subcarriers $\times 15 k H z \times 25 R B)$.

We have shown in our previous study [6] that $K \in[10,15]$ ( $K$ channel estimations) for 2 antennas are neccessary to estimate the calibration parameters, accordingly we initialize the number of required channel estimations to $K=10$. Then, assuming that the channel is constant during one frame duration $(10 \mathrm{~ms})$, we store one UL/DL channel estimate by frame ( $K$ frames) at eNB. This first step corresponds to relative calibration training phase (described in [9]) which is performed using all the 300 subcarriers.

The required throughput to feed the signal back from UE2 to eNB2 depends on the selected modulation and coding scheme, the number of antennas ( $2 \mathrm{ant}$ ), the quantization of the DL channel ( 8 bits), and the number of subcarriers (300).

The DL channel is estimated by UE2 in one subframe and this estimation is fedback to the eNB2, using the PUSCH. Accordingly the transmission of all the subcarrier complex coefficients using the PUSCH requires 9600 bits (see [8]) transmitted in 1 frame duration $(10 \mathrm{~ms})$. However in order to reduce the length of the transmitted feedback data, another idea which is under investigation suggests to decimate the channel estimation at UE2. The higher the decimation factor, the lower the required payload in PUSCH. Then at the eNB2 side, the values will be reconstructed by an oversampling technique using the previous and next channel coefficients.

\section{B. Numerical Results}

In the simulation, the results are obtained for different values of the relevant parameters (the pathloss in free space $P_{l s}$, the SNR, the number of frame, etc). Furthermore, the selected reciprocity-calibration method has been evaluated by the normalized mean squared error (MSE) of the reconstructed DL channel at eNB2 $\hat{\mathbf{G}}_{s s}$ and the estimated DL channel $\mathbf{G}_{s s}$ from both antennas of eNB2 at UE2. Considering a Rayleigh channel model, we firstly assume that the channel is perfectly reciprocal (see perfect case Fig. 5) and then we observe the case when we activate the decalibration filters in Fig. 3. Fig. 5 shows that even if the MSE of the perfect reciprocity case slightly outperforms the decalibration case, the error generated by the filters can be easily compensated by the calibration procedure, and the reconstruction MSE of the DL channel using only the calibration parameters and the UL channel is close to the perfect case. 
After this over-the-air calibration step, the impact of the proposed spatial IW interference avoidance technique from SUs to PUs has been evaluated. We compare the DL constellation of the PUs before and after the activation of the precoder at the eNB2. Then, we run the simulation with 500 frames, $K=10$

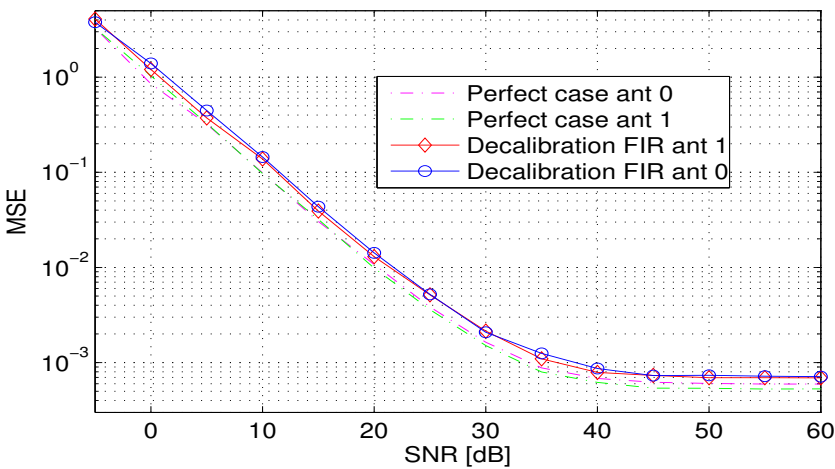

Fig. 5. MSE comparison of downlink channel reconstruction in the perfect case, then assuming the decalibration filters. The perfect case supposes that there are no perturbations from the RF front-ends.

and different MIMO channel models (extended vehicular A: EVA, spatial channel model C: SCM-C, etc), using the spatial IW precoder. In Fig. 6, we observe the constellations of the received signal at the primary receiver UE1 with 16QAM (quadrature amplitude modulation) with and without beamforming. Considering a low pathloss $P_{l s}=5 d B$ (which means that primary and secondary users are close e.g. in the same room), and a frequency selective Ricean channel model (at least one line of sight and 8 taps), we see that without a precoder at secondary, the interference from secondary leads to a bad constellation diagram at UE1. Subsequently, the precoder expressed in Section II is applied at eNB2. We observe the result assuming the same set of parameters. With the precoder applied at the secondary transmitter, the primary signal is not disturbed any more by the interference from eNB2, and the complex points of the constellation for 16QAM in Fig. 6 are well located in the diagram. It is clear that the precoder improves the UE1 signal reception in presence of interference from secondary. Eventually, using the ZFB, all systems are able to transmit, under the assumption that the determined calibration factors are constant during the process.

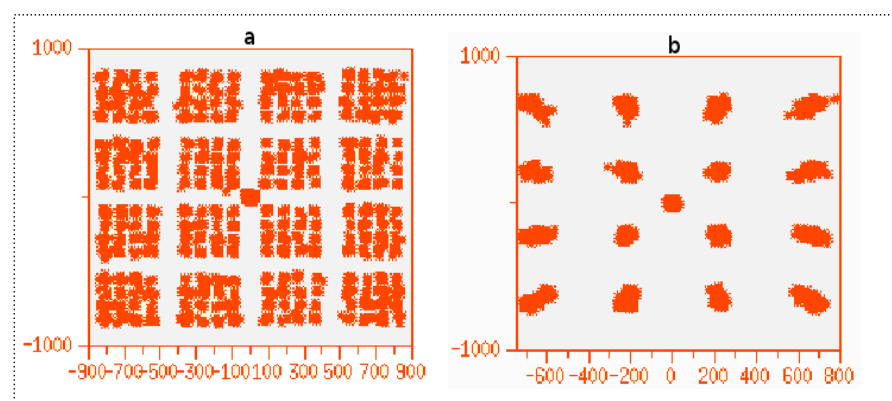

Fig. 6. The 16-QAM Constellation of primary receive signal at UE1, with interference from secondary eNB2 $P_{l s}=5 d B, S N R=25 d B$ in perfect reciprocity case, Fig. a: without $\mathrm{ZFB}$, and Fig. b: with ZFB activated.

A key assumption in this implementation is the TDD frame synchronization phase between eNB1 and eNB2. Our study has been achieved with the idea that eNB2 initially listens for the primary PSS every 100 frames. The synchronization has still to be implemented to allow a complete integration of the beamforming and calibration methods with the hardware.

\section{CONCLUSIONS}

In this paper, we propose a spatial interweave (IW) cognitive radio method and show that it's implementation is feasible on a real-time LTE platform.

We have proposed innovative solutions allowing to avoid interference towards the primary system. Specifically we designed, at the secondary base station (eNB2), an over-the-air calibration technique and a beamforming strategy based on the channel reciprocity hypothesis inherent in TDD systems. The spatial interweave CR has been performed without collaboration from the primary. The proposed spatial IW method has been implemented and integrated into the OpenAirInterface software modem. We presented results from simulations, which assess the good performance of the algorithms.

\section{REFERENCES}

[1] S. Haykin, "Cognitive radio: brain-empowered wireless communications," IEEE Journ. Selected Areas in Communications, vol. 23, no. 2, pp. 201-220, 2005.

[2] A. Goldsmith, S.A. Jafar, I. Maric, and S. Srinivasa, "Breaking spectrum gridlock with cognitive radios: An information theoretic perspective," Proceedings of the IEEE, vol. 97, no. 5, pp. 894-914, 2009.

[3] F. Negro, I. Ghauri, and D.T.M. Slock, "Transmission techniques and channel estimation for Spatial Interweave TDD Cognitive Radio systems," in 43rd IEEE Asilomar Conf. 2009, pp. 523-527.

[4] S. Filin, H. Harada, H. Murakami, and K. Ishizu, "International standardization of cognitive radio systems," Communications Magazine, IEEE, vol. 49, no. 3, pp. 82-89, 2011.

[5] P. Pawelczak, K. Nolan, L. Doyle, S.W. Oh, and D. Cabric, "Cognitive radio: ten years of experimentation and development," Communications Magazine, IEEE, vol. 49, no. 3, pp. 90-100, 2011.

[6] B. Kouassi, I. Ghauri, B. Zayen, and L. Deneire, "On the performance of calibration techniques for cognitive radio systems," in The 14th International Symp. WPMC, France, 2011.

[7] B.D. Van Veen and K.M. Buckley, "Beamforming: A versatile approach to spatial filtering," ASSP Magazine, IEEE, vol. 5, no. 2, pp. 4-24, 1988.

[8] B. Zayen, B. Kouassi, R. Knopp, F. Kaltenberger, D. Slock, I. Ghauri, and L. Deneire, "Software implementation of spatial interweave cognitive radio communication using OpenAirInterface platform," in The International Symp. ISWCS, France, 2012.

[9] M. Guillaud, D.T.M. Slock, and R. Knopp, "A practical method for wireless channel reciprocity exploitation through relative calibration," 8th ISSPA, Australia, pp. 403-406, 2005.

[10] I. Markovsky and S. Van Huffel, "Overview of total least-squares methods," Signal processing, vol. 87, no. 10, pp. 2283-2302, 2007.

[11] M. Guillaud and F. Kaltenberger, "Towards practical channel reciprocity exploitation: Relative calibration in the presence of frequency offset," in IEEE WCNC Conf. on, Shanghai, China, 2013.

[12] B. Kouassi, B. Zayen I. Ghauri, and L. Deneire, "Reciprocity calibration techniques, implementation on the OpenAirInterface platform," in 4th International Conf. on Cognitive Radio and Advanced Spectrum Management (COGART), Spain, 2011.

[13] F. Kaltenberger, H. Jiang, M. Guillaud, and R. Knopp, "Relative channel reciprocity calibration in MIMO/TDD systems," in IEEE Future Network and Mobile Summit, 2010, pp. 1-10.

[14] R. Rogalin, O.Y. Bursalioglu, H.C. Papadopoulos, G. Caire, and A.F. Molisch, "Hardware-impairment compensation for enabling distributed large-scale mimo," in Proc. ITA Workshop, San Diego, CA, USA, 2013.

[15] S. Sesia, I. Toufik, and M. Baker, LTE, The UMTS Long Term Evolution: From Theory to Practice, John Wiley \& Sons Inc, 2009. 


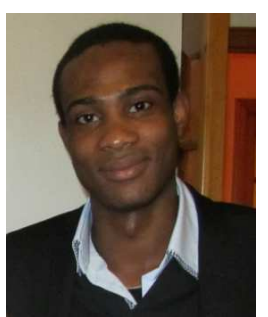

Boris Kouassi (kouassi@i3s.unice.fr) received in 2009 the Eng. degree in Telecommunication and Computer Network at the Engineering school of Casablanca ISERT, Morocco. He earned in 2010 the M.Sc. degree at PHELMA, the Engineering school of Physics, Applied Physics, Electronics and Materials Science of Grenoble Institute of Technology (INP), France.

$\mathrm{He}$ is currently pursuing the Ph.D. degree (since 2010) in Signal Processing and Telecommunication in the I3S laboratory at the University of Nice Sophia-Antipolis (France), in collaboration with Intel Mobile Communications. His research centers around Cognitive Wireless Communication techniques and Signal Processing in a multi-user MIMO environment.

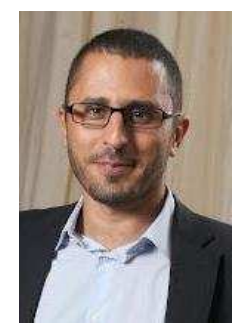

Bassem Zayen (zayen@eurecom.fr) was born in Sfax, Tunisia, in 1980. He received the Eng. degree in Telecommunications at ENIT in 2004 and the M.Sc degree in communication systems in 2005 . $\mathrm{He}$ was a member in the research unit Signaux et Systèmes at ENIT and teaches courses in Telecommunications from 2005 to 2007. In 2010, he obtained the Ph.D. degree in electronic and communications from TELECOM ParisTech.

During his doctoral studies at EURECOM, he worked on spectrum sensing and resource allocation for cognitive radio systems. He returned to EURECOM's Mobile Communications group from February 2011 to July 2012 as a postdoc. He was working in real-time platform architectures and experimentation in cognitive radio systems and cellular technologies (LTE, LTEAdvanced). From August 2012, he is working at Intel Mobile Communications, France, in software radio architectures and LTE modem algorithms integration.

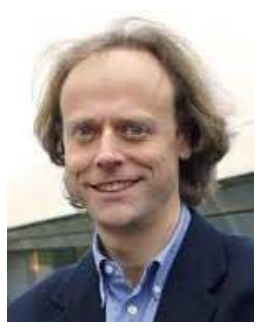

Raymond Knopp (knopp@eurecom.fr) received the B.Eng. (Honours) and the M.Eng. degrees in electrical engineering from McGill University, Montreal, Canada, in 1992 and 1993, respectively. In 1997, received the $\mathrm{PhD}$ degree in communication systems from the Swiss Federal Institute of Technology, Lausanne (EPFL). During his Ph.D. studies (19931997), he was a Research and Teaching Assistant in the Mobile Communications Department of EURECOM, Sophia-Antipolis, France. From 19972000 he was a research associate in the Mobile Communications Laboratory of EPFL. In 2000 he rejoined the Mobile Communications Department of EURECOM as a Professor. His current research and teaching interests are in the area of digital communications, software radio architectures, and implementation aspects of signal processing systems. He has published numerous journal and conference articles and he is actively involved in many collaborative research projects in wireless communication systems with industry

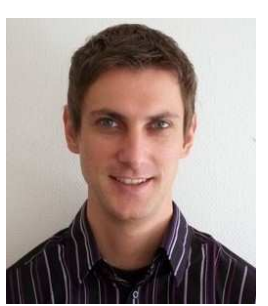

Florian Kaltenberger (kaltenberger@eurecom.fr) (S'05-M'08) received his Dipl.-Ing. degree and his $\mathrm{Ph} . \mathrm{D}$. degree both in technical mathematics from the Vienna University of Technology in 2002 and 2007, respectively. He is an assistant professor at the Mobile Communications Department of EURECOM, Sophia-Antipolis, France, where he supervises activities around the real-time open-source experimental platform OpenAirInterface.org. From 2003 to 2007 he was with the Wireless Communications Group of the Austrian Research Centers, where he was developing a real-time MIMO channel emulator. His research interests focus on practical aspects of wireless communications systems (especially LTE), such as transceiver design and implementation, efficient exploitation of feedback in MIMO systems, radio resource management, experimental performance analysis, and scalable system simulation and modeling.

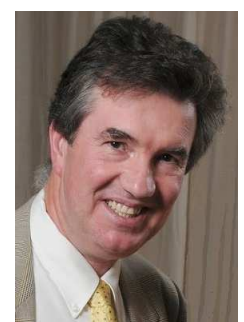

Dirk T.M. Slock (dirk.slock@eurecom.fr) received an Eng. degree from the University of Gent, Belgium in 1982. In 1984 he was awarded a Fulbright scholarship for Stanford University, USA, where he received the MSEE, MS in Statistics, and $\mathrm{PhD}$ in $\mathrm{EE}$ in 1986, 1989 and 1989 resp. In 1989-91, he was a member of the research staff at the Philips Research Laboratory Belgium. In 1991, he joined EURECOM where he is now professor and teaches statistical signal processing (SSP) and signal processing (SP) for wireless communications. His research interests include SSP for mobile communications and audio processing. He invented semi-blind channel estimation, the chip equalizer-correlator receiver used by 3G HSDPA terminals, spatial multiplexing cyclic delay diversity now part of LTE, and his work led to the Single Antenna Interference Cancellation concept used in GSM terminals. Recent keywords are diversity-multiplexing tradeoff, MIMO interference channel, variational Bayesian techniques, large system analysis, audio source separation. He is the (co)author of around 400 articles and received a best journal paper award from IEEE-SP and from EURASIP. $\mathrm{He}$ is the coauthor of two IEEE Globecom'98, one IEEE SIU'04 and one IEEE SPAWC' 05 best student paper award, and a honorary mention at IEEE SSP'05, IWAENC'06 and IEEE Asilomar'06. He was an associate editor for the IEEE-SP Trans. in 1994-96 and the IEEE SP Letters in 2009-10. He is an editor for the EURASIP Journ. on Advances in SP (JASP). He is a member of the IEEE-SPS Awards Board, the EURASIP JWCN Awards Committee and was the General Chair of the IEEE-SP SPAWC'06 workshop.

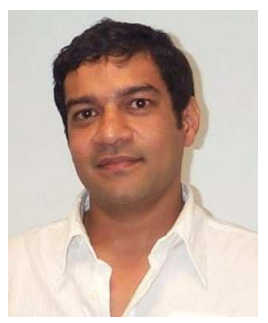

Irfan Ghauri (irfan.ghauri@intel.com) received the Ph.D. degree in Signal Processing at EURECOM, Sophia-Antipolis, France, in 2000. He is a Senior Member of Technical Staff at Wireless Research and Development facility of Intel Mobile Communications, Sophia Antipolis, where he manages research activities in partnership with academic institutions. He was Member of Technical Staff at Ericsson Mobile Communications, Sweden, technical lead at 3G.com UK Ltd., Bath, UK, and Vice-President at Cellular3G Inc.. His research interests range from multi-user communication theory, signal processing (SP) for communications, interference suppression and management in wireless networks. His best known work is the co-invention of UMTS downlink chip equalizer receiver in 1998, which has since become the standard 3G+ handset receiver. He is Associate Member of the IEEE-SP Society's SP for Communications and Networking Technical Committee and represents Intel France in several European projects. He has supervised over ten M.Sc. and five Ph.D. theses on various topics and teaches at graduate level.

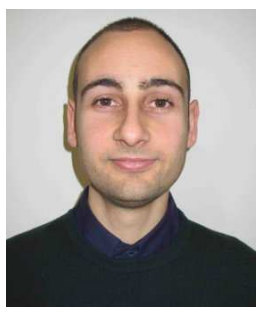

Francesco Negro (negro@eurecom.fr) received his B.S. from University of Lecce (Italy) in 2006 and in 2008 his M.Sc. from Politecnico di Torino (Italy) both in Telecommunication Engineering.

From May 2009, he has joined EURECOM (France) as a $\mathrm{PhD}$ student. His main research activities are on signal processing for wireless communications with particular interest in transmitter and receiver design for Interference Channel and Cognitive Radio.

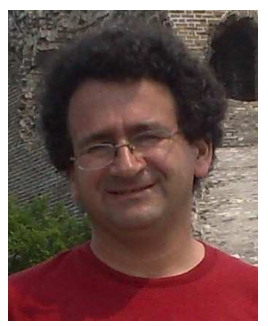

Luc Deneire (luc.deneire@unice.fr) received the Eng. degree in Electronics from University of Liege (Belgium) in 1988, the Eng. degree in Telecommunications from University of Louvain-La-Neuve in 1994 and the PhD degree in Signal Processing at EURECOM, Sophia-Antipolis, France in 1998. He has been working for IMEC, Leuven, Belgium from 1999 to 2002. Since 2002, he is Professor at University of Nice Sophia-Antipolis, currently in the Polytechnic School of the University, where he is heading the EE department. He is member of the I3S laboratory. He is working on the design of wireless communications networks. His main interests are on the signal processing algorithms involved, as well as on the cross-layers aspects of mobile and cognitive networks. 